\title{
A condição de altitude simulada piora o estado de humor e aumenta a pressão arterial sistólica de jovens saudáveis
}

\author{
The simulated altitude condition worsens mood and increases systolic \\ blood pressure in healthy young
}

Jorge Fernando Tavares de Souza ${ }^{1,2}$, Sara Quaglia de Campos Giampá1,2, Valdir de Aquino Lemos $^{1,2}$, Ronaldo Vagner Thomatielli dos Santos ${ }^{1,2}$, Marco Túlio de Mello ${ }^{3}$, Hanna Karen Moreira Antunes ${ }^{1,2^{*}}$

ARTIGO ORIGINAL | ORIGINAL ARTICLE

\begin{abstract}
RESUMO
A literatura aponta que o estresse do ambiente aliado ao estresse do exercício físico produz alterações psicológicas e fisiológicas. Portanto, o objetivo do presente estudo foi investigar a associação entre o efeito agudo do exercício físico moderado realizado em condição de atitude simulada sobre o estado de humor, saturação da oxihemoglobina e pressão arterial de jovens saudáveis. Dez voluntários realizaram $45 \mathrm{~min}$. de exercício físico e passaram por duas condições: Condição Normóxia $(\mathrm{CN})$ e Condição Hipóxia $(\mathrm{CH})$. Em ambas as condições eles responderam a dois instrumentos que avaliam as respostas de humor, a Escala de Humor de Brunel (BRUMS) e a Escala Subjetiva de Experiência em Exercício (SEES), bem como a avaliação da saturação de oxihemoglobina e da pressão arterial. Esses procedimentos foram realizados antes, imediatamente após, $30 \mathrm{~min}$. e $60 \mathrm{~min}$. após o término do protocolo. Após a prática do exercício físico na Condição Hipóxia, os voluntários apresentaram maiores escores de Fadiga, Confusão Mental, DTH, Raiva, Distresse Psicológico e menores valores de Vigor e Bem-Estar Positivo acompanhados de uma diminuição da saturação da oxihemoglobina. O exercício físico moderado realizado em altitude simulada de $4500 \mathrm{~m}$ promove mudanças no estado de humor e aumento da pressão arterial sistólica de jovens saudáveis.

Palavras-chave: exercício físico, hipóxia, estado de humor, respostas psicobiológicas e respostas fisiológicas
\end{abstract}

ABSTRACT

The literature suggests that stress the environment coupled with the stress of exercise produces psychological and physiological changes. Therefore, the aim of this study was to investigate the association between the acute effect of moderate physical exercise performed in conditions of simulated attitude about the mood, oxyhemoglobin saturation and blood pressure in healthy young adults. Ten volunteers performed 45 min exercise and have gone through two conditions: Condition normoxic $(\mathrm{CN})$ and Condition hypoxia $(\mathrm{CH})$. In both conditions they responded to two instruments that assess the responses of humor, the Brunel Mood Scale (BRUMS) and the Scale of Subjective Experience in Exercise (SEES), as well as evaluating the oxyhemoglobin saturation and blood pressure. These procedures were performed before, immediately after, $30 \mathrm{~min}$ and $60 \mathrm{~min}$ after the protocol. After physical exercise in hypoxia condition, the volunteers had higher scores Fatigue, Mental Confusion, DTH, Anger, Psychological distress and lower levels of force and Wellness Positive accompanied by a decrease in oxyhemoglobin saturation. Moderate physical exercise performed at a simulated altitude of $4500 \mathrm{~m}$ promotes changes in mood and increased systolic blood pressure in healthy young adults.

Keywords: exercise, hypoxia, mood, psychobiological responses and physiological responses

\footnotetext{
Artigo recebido a 25.06.2014; Aceite a 13.01.2015

${ }^{1}$ Departamento de Biociências, Universidade Federal de São Paulo- UNIFESP, Campus Baixada Santista, Brasil

${ }^{2}$ Centro de Estudos em Psicobiologia e Exercício - CEPE, Brasil

${ }^{3}$ Departamento de Educação Física, Universidade Federal de Minas Gerais - UFMG, Brasil

* Autor correspondente: Rua Silva Jardim, 136 Vila Mathias, Santos - SP, Brasil. Código Postal: 11015-020.

E-mail: hanna.karen@unifesp.br
} 


\section{INTRODUÇÃO}

Atualmente por diversas razões - incluindo o turismo, lazer, questões profissionais - o número de pessoas expostas a elevadas altitudes vem crescendo a cada ano (Sharma et al., 2014), assim como o número de estudos referente às alterações fisiológicas (Born et al., 2014) e do comportamento humano (Lemos et al., 2010) observadas em tal condição.

O exercício físico, enquanto agente estressor, quando realizado em locais elevados sofre grande influencia da hipóxia que é a diminuição da pressão barométrica e da pressão parcial de oxigênio, culminando em uma dificuldade na utilização do aporte de oxigênio (Peng et al., 2013). As grandes altitudes induzem a hipóxia, pois quanto maior a altitude, menores são as concentrações de oxigênio no sangue arterial. Isso pode impactar diretamente no desempenho, na capacidade máxima de exercício, no tempo de resistência e na fadiga geral (Chapman, Stickford, \& Levine, 2010; Mazzeo, 2005). A prática do exercício físico em ambientes elevados promove mudanças tanto fisiológicas quanto psicológicas (Ando, Yamada, \& Kokubu, 2010; Subudhi, Dimmen, \& Roach, 2007; Subudhi, Miramon, Granger, \& Roach, 2009).

Acredita-se que a hipóxia seja responsável pelo início de uma cascata de eventos sinalizadores que levam à adaptação à altitude. Entre esses eventos, destacam-se alterações que são produzidas no sistema cardiovascular, musculoesquelético, endócrino, imune e assim chegando até o cérebro (Virués-Ortega, Garrido, Javierre, \& Kloezeman, 2006). Esses ajustes buscam a homeostasia do organismo, porém o indivíduo sofre com a sonolência, a fadiga mental e muscular e prostração. E durante esse processo de adaptação há uma diminuição do desempenho físico tanto máximo quanto submáximo (Araújo, 2009).

Do ponto de vista psicológico, a ascensão a grandes altitudes é capaz de produzir uma gama de alterações, notadamente nas funções cognitivas e no estado de humor. Lemos e colaboradores (de Aquino Lemos et al., 2012) mostraram que indivíduos expostos a uma condição de altitude simulada o equivalente a 4500m apresentaram diminuição dos níveis de atenção, concentração e velocidade de processamento mental, assim como prejuízos visuais e na memória de trabalho e maior dificuldade em realizar funções executivas. Além disto, também foi observada uma piora no estado de humor, incluindo o vigor, aliado a um aumento de sintomas depressivos, raiva, fadiga e irritabilidade (Bahrke \& Shukitt-Hale, 1993; de Aquino Lemos et al., 2012). A magnitude dessas alterações dependem da altitude, do tempo de exposição e de ascensão, além do período de adaptação de cada indivíduo (Ando et al., 2013).

Diante deste contexto, acredita-se que a realização aguda do exercício físico moderado em condição hipóxica seja o gatilho para alterar parâmetros fisiológicos, como o aumento da pressão arterial, e parâmetros psicológicos, como a piora do estado de humor. Segundo Mannie et al. (2013) é possível que a vulnerabilidade a doenças como hipertensão arterial esteja associada a alterações do estado de humor, por exemplo a depressão. Além disso, Pointer, Livingston, Yancey, McClelland, e Bukoski (2008) mostraram que a raiva também faz parte do estado de humor e pode apresentar relações com a pressão arterial.

Sendo assim, o objetivo do presente estudo foi investigar os efeitos agudos do exercício físico moderado realizado em condição de atitude simulada sobre a saturação da oxihemoglobina, a pressão arterial e o estado de humor de jovens saudáveis.

\section{MÉTODo}

\section{Amostra}

Foram selecionados 10 voluntários do sexo masculino, fisicamente ativos, não apresentavam doenças clinicamente diagnosticadas, estudantes universitários, cujas características são apresentadas em média \pm desvio padrão (idade $22.30 \pm 2.79$ anos, Estatura $176 \pm 0.04 \mathrm{~cm}$, Massa Corporal 72.66 $\pm 11.86 \mathrm{~kg}$, IMC $\left.23.37 \pm 3.74 \mathrm{~kg} / \mathrm{m}^{2}\right)$. Os mesmos auto-relataram não fazerem uso de tabaco, álcool, drogas ilícitas e/ ou medicamentos controlados que poderiam ou não alterar o estado de humor. Todos os 
voluntários foram submetidos a 2 condições distintas: Condição Normóxia $(\mathrm{CN})$ e Condição Hipóxia $(\mathrm{CH})$.

\section{Instrumentos}

Simulador de altitudes. Câmara normobárica (CAT - Colorado Altitude Training ${ }^{\mathrm{TM}} /$ CAT-12 Air Unit). O estudo foi realizado em uma câmara equipada para simulações de altitude que varia até $4,500 \mathrm{~m}$, o que equivale a uma pressão barométrica de 433 mmHg e uma fração inspirada de oxigênio (FiO2) de 13,5\% $\mathrm{O}_{2}$.

\section{Avaliações objetivas}

Exercício físico.

O exercício físico foi realizado em uma esteira ergométrica (Lifefitness ${ }^{\circledR}$ 9700HR, Schiller Park, IL, USA).

\section{Ergoespirometria.}

A avaliação ergoespirométrica foi realizada por meio do Quark, PFT - Pulmonary Function Testing - FRC \& DLCO - 4Ergo, Cosmed, Italy. Este sistema foi calibrado antes da realização de cada protocolo, usando uma concentração de gases conhecidos, sendo que as calibrações do volume e do fluxo foram realizadas com o auxílio de uma seringa de três litros. Uma máscara facial Hans Rudolph ${ }^{\circledR}$ flow-by face mask (Kansas City, MO, EUA), de silicone para maior conforto dos voluntários foi utilizada.

\section{Monitoração da freqüência cardíaca.}

As monitorações foram realizadas por meio de um cardiofreqüencímetro (Polar ${ }^{\circledR}$, modelo FS1, Kempele, Finland).

\section{Monitoração da saturação de oxigênio.}

As monitorações foram realizadas por meio de um oximetro de pulso (Fingertip Pulse modelo MD300C202).

\section{Monitoração da pressão arterial.}

As monitorações foram realizadas por meio de um estetoscópio e um esfigmomanômetro.

\section{Avaliações subjetivas \\ Perfil de humor.}

Escala de Humor de Brunel (BRUMS) Desenvolvida para medir rapidamente o estado de humor (Terry, Lane, \& Fogarty, 2003) e validado para a língua portuguesa por Rohlfs et al. (2008), foi adaptado do "Profile of Mood States (POMS) (McNair, Lorr, \& Droppleman, 1971). Consiste em uma lista com 24 adjetivos relacionados ao estado de humor, onde o avaliado deve anotar como se sente em relação a cada adjetivo, conforme as instruções considerando uma escala tipo Likert de 0 a 4 . Seis fatores de humor ou estados afetivos são medidos por esse instrumento: tensão, depressão, raiva, vigor, fadiga e confusão. É esperado nesse teste que os valores encontrados para a dimensão vigor sejam maiores que os valores apresentados nas outras dimensões, o que denotaria um perfil de humor em forma de "Iceberg". Para o Distúrbio Total de Humor (DTH) os valores são computados e calculados pela soma das cinco variáveis negativas, subtraindo a pontuação da dimensão vigor. Nesta dimensão, scores mais altos indicam piora no estado de humor.

Respostas afetivas.

Escala Subjetiva de Experiência em Exercício (SEES) - Instrumento utilizado para observar respostas afetivas induzidas pelo exercício físico. Trata-se de uma escala tridimensional, que avalia as seguintes dimensões: (a) bem-estar positivo; (b) distresse psicológico e (c) fadiga. A principal questão é: "Como você se sente agora?", a escala é composta por 12 itens graduados em uma escala tipo Likert que varia de 1 a 7 , onde 1 significa nenhum pouco e 7 significa muitíssimo (Lox \& Rudolph, 1994; McAuley \& Courneya, 1994).

\section{Procedimentos}

Antes de participarem do presente estudo, todos os possíveis riscos envolvidos no procedimento experimental foram explicados aos voluntários e a sua participação foi por livre e espontânea vontade. O estudo foi aprovado pelo Comitê de Ética em Pesquisa da 
Universidade Federal de São Paulo/Hospital São Paulo (0620/09).

O delineamento do estudo foi ensaio clínico cross over cego para os voluntários. Os mesmos foram mantidos em condição de hipóxia equivalente a $4500 \mathrm{~m}$ de altitude ou normóxia durante 90 minutos.

Os experimentos foram realizados em três dias não consecutivos, com intervalo de uma semana entre cada experimento. Em uma primeira visita, os voluntários foram submetidos a uma avaliação clínica, por meio de um Eletrocardiograma de repouso (ECG) e a um Teste Ergométrico com o médico do laboratório. Após a realização destas avaliações, aqueles considerados aptos pelo médico, participaram do estudo. Em uma segunda visita, os voluntários foram submetidos a um Teste Ergoespirométrico utilizando um protocolo de cargas progressivas até a exaustão voluntária máxima. Este teste foi realizado para determinar a intensidade do Limiar Ventilatório 1 (LV-I). Este protocolo consistiu em uma velocidade de $1 \mathrm{~km} / \mathrm{h}$ a cada minuto, sendo a carga inicial para aquecimento de três minutos a $6 \mathrm{~km} / \mathrm{h}$. O teste foi encerrado quando o voluntário atingiu a exaustão voluntária máxima. Essa exaustão foi definida como a inabilidade em acompanhar a velocidade da esteira por 15 segundos, ou até que os voluntários solicitassem a parada do teste mesmo sendo encorajados a continuar (Walsh, Gonzalez, Ratamess, Kang, \& Hoffman, 2010). Durante todo o teste foi utilizada uma inclinação fixa de $1 \%$ para simular o desgaste físico em locais abertos (Jones \& Doust, 1996).

Posteriormente, o exercício físico com carga retangular foi realizado dentro da câmara normobárica tanto na $\mathrm{CN}$ quanto na $\mathrm{CH}$ na mesma esteira do teste ergoespirométrico, porém na intensidade individual do LV-I e realizados por um período de 45 minutos, representando $50 \%$ a mais do volume (tempo) de acordo com o American College of Sports Medicine (Nelson et al., 2007). O protocolo adotado consistiu de aquecimento por 3 minutos a $7 \mathrm{~km} / \mathrm{h}$, com imediato ajuste a carga individual do LV-I sendo esta intensidade mantida por 45 minutos de forma ininterrupta. Este protocolo foi diferente apenas na altitude simulada, sendo que, a partir de 15 minutos de exercício físico houve uma redução da intensidade da esteira para a velocidade de aquecimento, isto é, para $7 \mathrm{~km} / \mathrm{h}$, que foi mantida até o término do exercício físico, o que representa uma redução de aproximadamente $30 \%$ da intensidade do esforço. Esta diminuição na intensidade do esforço se deve ao fato de os voluntários não conseguirem manter a velocidade do LV-I em função do esforço gerado pelo exercício e pela hipóxia. Diante disso, por uma questão de segurança frente ao esforço do exercício físico associado aos efeitos da hipóxia, a velocidade da esteira foi reduzida. Além disso, colocamos esse fator como uma das limitações em nosso estudo.

Durante todos os testes, a monitoração contínua da frequência cardíaca foi realizada. $\mathrm{O}$ experimento da $\mathrm{CH}$ foi realizado com intervalo de uma semana após o da $\mathrm{CN}$, iniciando no período da tarde por volta das 15 horas e finalizando às 16:30 horas.

A avaliação do perfil de humor e das respostas afetivas, bem como a mensuração da saturação da oxihemoglobina e da pressão arterial foram realizadas em 4 momentos: basal, imediatamente após, 30 e 60 minutos após o término dos protocolos. Após os voluntários completaram o protocolo experimental, o simulador de altitude foi desligado e os voluntários foram dispensados quando não exibiam quaisquer sintomas clínicos que impediam sua saída do laboratório.

\section{Análise estatística}

Os dados são descritos como média \pm desvio-padrão (DP). A normalidade dos dados foi verificada por meio do teste Shapiro Wilk's. Para comparar as medidas de dados paramétricos foi realizada uma análise de variância ANOVA para medidas repetidas, seguida do teste de post-hoc de Duncan. O nível de significância adotado foi de $p \leq 0.05$. As análises estatísticas foram realizadas utilizando o software Statistica $^{\circledR}$ versão 7.0. (http://www.statsoft.com). 


\section{RESULTADOS}

A tabela 1 apresentada os dados referentes à análise descritiva da amostra referentes a idade, estatura, massa corporal e índice de massa corporal, bem como os resultados do teste ergoespirométrico realizado até a exaustão voluntária máxima no momento basal. Os parâmetros referentes ao consumo de oxigênio são apresentados em valores absolutos (litros/minuto) e em valores relativos (considerando a massa corporal total). Além disso, são apresentados os valores máximos da velocidade, da frequência cardíaca e do tempo.

Tabela 1

Dados Descritivos da Amostra

\begin{tabular}{|c|c|}
\hline Variáveis & Media \pm Desvio padrão \\
\hline Idade (anos) & $22.30 \pm 2.79$ \\
\hline Estatura (m) & $1.76 \pm 0.04$ \\
\hline Massa Corporal (kg) & $72.66 \pm 11.86$ \\
\hline $\operatorname{IMC}\left(\mathrm{kg} / \mathrm{m}^{2}\right)$ & $23.37 \pm 3.74$ \\
\hline$\dot{\mathrm{V}}_{2}$ pico (L.min) & $3.53 \pm 0.45$ \\
\hline$\dot{\mathrm{V}} \mathrm{O}_{2}$ pico $\left(\mathrm{mL} \cdot \mathrm{kg} \cdot \mathrm{min}^{-1}\right)$ & $49.84 \pm 6.01$ \\
\hline FC Max (bpm) & $190.90 \pm 8.49$ \\
\hline Velocidade Máx. (Km.h) & $15.80 \pm 1.40$ \\
\hline Tempo Max. (min) & $12.46 \pm 1.51$ \\
\hline \multicolumn{2}{|c|}{$\begin{array}{l}\text { Nota: Dados apresentados média } \pm \text { desvio padrão } \\
\text { referentes a } 10 \text { voluntários em cada grupo. } \\
\text { Legenda: IMC- índice de massa corporal; } \mathrm{V}_{2} \text { - consumo de } \\
\text { oxigênio; FC- freqüência cardíaca. }\end{array}$} \\
\hline
\end{tabular}

A tabela 2 mostra os resultados do questionário BRUMS e suas dimensões: tensãoansiedade, depressão, raiva-hostilidade, vigor, fadiga, confusão mental e DTH. Na comparação entre os grupos, não foi encontrada diferença significativa. Contudo, na comparação do comportamento das dimensões em relação ao tempo, observou-se que a dimensão Depressão $\left[\mathrm{F}_{(1,54)}=3.02 ; p<0.03\right]$ na condição normóxia apresentou um aumento $30 \mathrm{~min}$. após o término do exercício físico comparado com o basal, retornando aos valores iniciais após 60 min. $\mathrm{Na}$ dimensão Fadiga $\left[\mathrm{F}_{(1,54)=} 15.68\right.$; $p<0.0001]$ encontrou-se um aumento em relação ao basal no momento imediatamente após, comportamento similar encontrado na dimensão Confusão Mental $\left[\mathrm{F}_{(1,54)}=9.32\right.$; $p<0.0001] \quad$ e no DTH $\left[\mathrm{F}_{(1,54)=}=10.26\right.$; $p<0.0001]$.

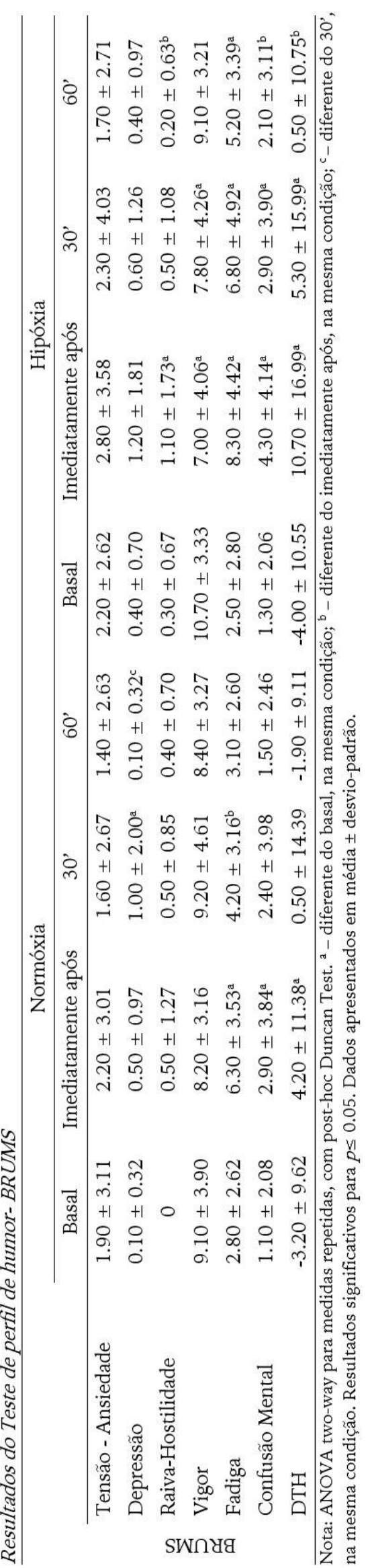


Para a condição hipóxia, observou-se que a dimensão Raiva aumentou no momento imediatamente após comparado ao basal, seguido de diminuição desses escores $60 \mathrm{~min}$. após o termino do protocolo de exercício físico. $\mathrm{Na}$ dimensão Vigor, encontrou-se diminuição no momento imediatamente após a realização do exercício que perdurou até $30 \mathrm{~min}$. $\mathrm{Na}$ dimensão Fadiga, encontramos aumento desses escores em todos os momentos pós-exercício físico. Na dimensão Confusão Mental e o DTH apresentaram comportamentos similares, onde encontramos um aumento dos escores imediatamente após a realização do exercício que perdurou até $30 \mathrm{~min}$. As outras variáveis não demonstraram diferenças significativas.

A figura 1 mostra os resultados do SEES incluindo bem-estar positivo, distresse psicológico e fadiga. Na condição normóxia é observado um aumento da dimensão Distresse Psicológico e Fadiga na condição imediatamente após, sendo que a Fadiga diminuiu esses escores 30 e $60 \mathrm{~min}$. após o termino do exercício físico. $\mathrm{Na}$ condição hipóxia, a dimensão Bem-estar Positivo apresentou redução desses escores no momento imediatamente após perdurando até $30 \mathrm{~min}$. do término do exercício físico. $\mathrm{Na}$ dimensão Distresse Psicológico apresentou um aumento no momento imediatamente após perdurando até 30 min., e a dimensão Fadiga apresentou aumento dos escores imediatamente após perdurando até $30 \mathrm{~min}$., mostrando recuperação $60 \mathrm{~min}$. após o término do protocolo de exercício físico. As outras variáveis não demonstraram diferenças significativas.

A figura 2 apresenta os dados da saturação da oxihemoglobina a partir da oximetria de pulso. Na condição normóxia não foi observado diferenças significativas, no entanto, na condição de hipóxia é possível observar que imediatamente após houve uma diminuição deste parâmetro que perdurou por até $30 \mathrm{~min}$. Além disso, observou-se que no momento 60 min. houve uma recuperação desta variável. A comparação inter-grupo demonstrou que o momento imediatamente após e $30 \mathrm{~min}$. são diferentes onde a condição normóxia demonstrou maiores porcentagens de saturação de oxigênio quando comparado com a condição de hipóxia.
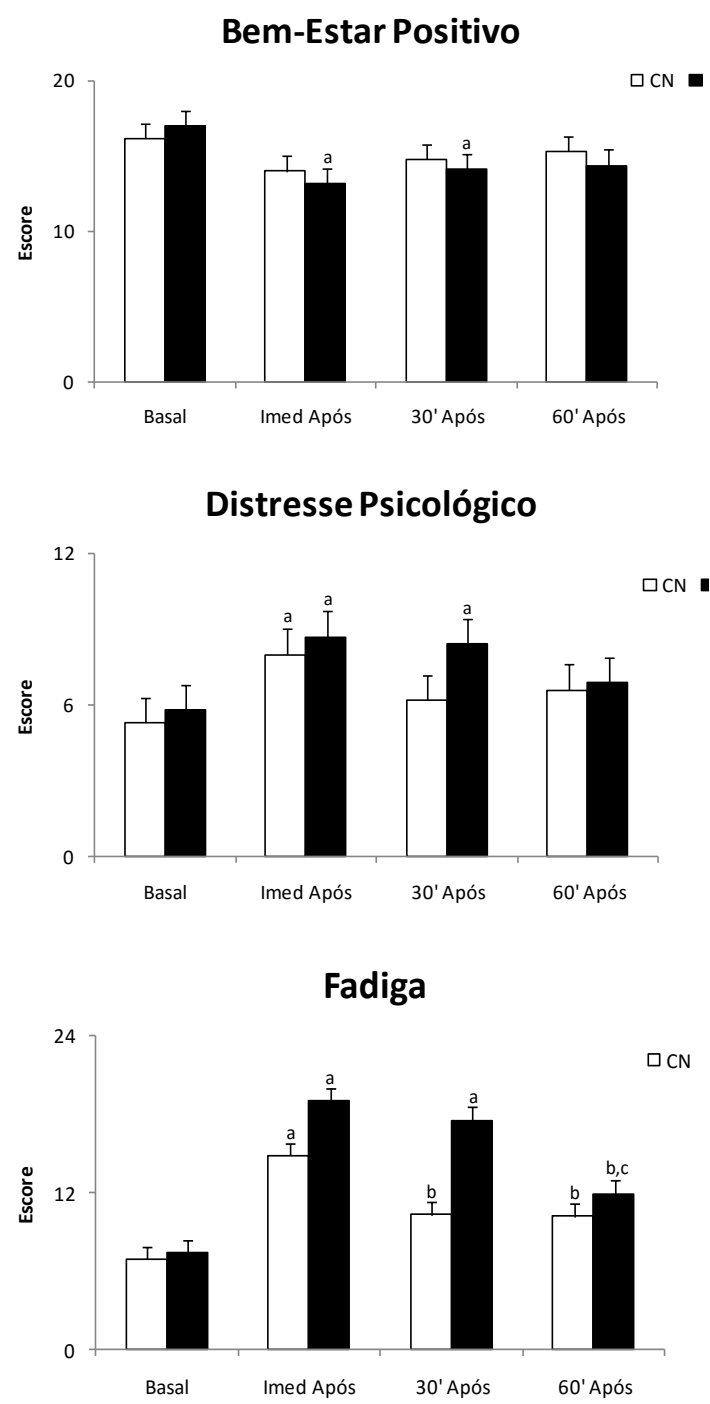

Figura 1. Resultados da Escala Subjetiva de Experiência em Exercício- SEES. Nota: ANOVA twoway para medidas repetidas, com post-hoc utilizando Duncan Test. ${ }^{\mathrm{a}}$ - diferente do basal, na mesma condição; ${ }^{\mathrm{b}}$ - diferente do imediatamente após, na mesma condição; ${ }^{c}$ diferente do 30', na mesma condição. Resultados significativos para $p \leq 0.05$. Dados apresentados em média \pm desvio-padrão. Dados referentes a 10 voluntários.

A figura 3 apresenta os dados da pressão arterial. Em relação a pressão arterial sistólica, encontrou-se um aumento desta variável imediatamente após na condição normóxia, sendo esse comportamento similar ao da condição hipóxia. No entanto, nessa condição foi observado uma diminuição dos valores nos momentos 30 e 60 min. após a prática do exercício. As outras variáveis não demonstraram diferenças significativas. 


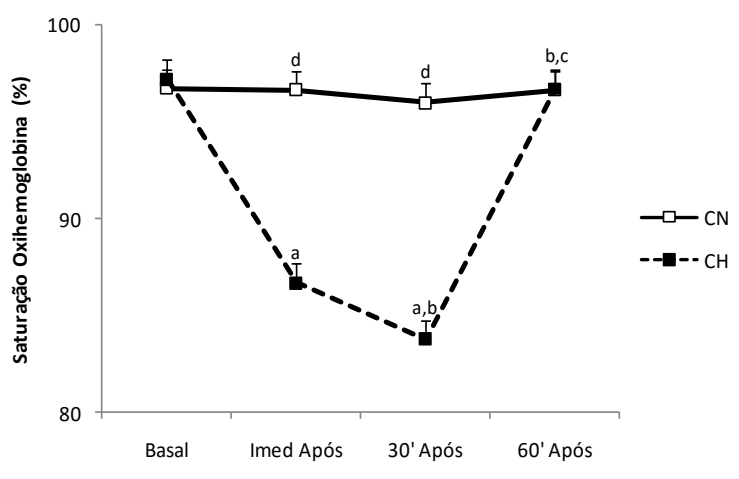

Figura 2 - Resultados da Saturação da Oxihemoglobina. Nota: ANOVA two-way para medidas repetidas, com post-hoc utilizando Duncan Test. ${ }^{a}$ diferente do basal, na mesma condição; ${ }^{\mathrm{b}}$ - diferente do imediatamente após, na mesma condição; ${ }^{\mathrm{c}}$ - diferente do 30', na mesma condição; ${ }^{d}$ - diferente intergrupo, no mesmo instante. Resultados significativos para $p \leq 0.05$. Dados apresentados em média \pm desvio-padrão. Dados referentes a 10 voluntários.
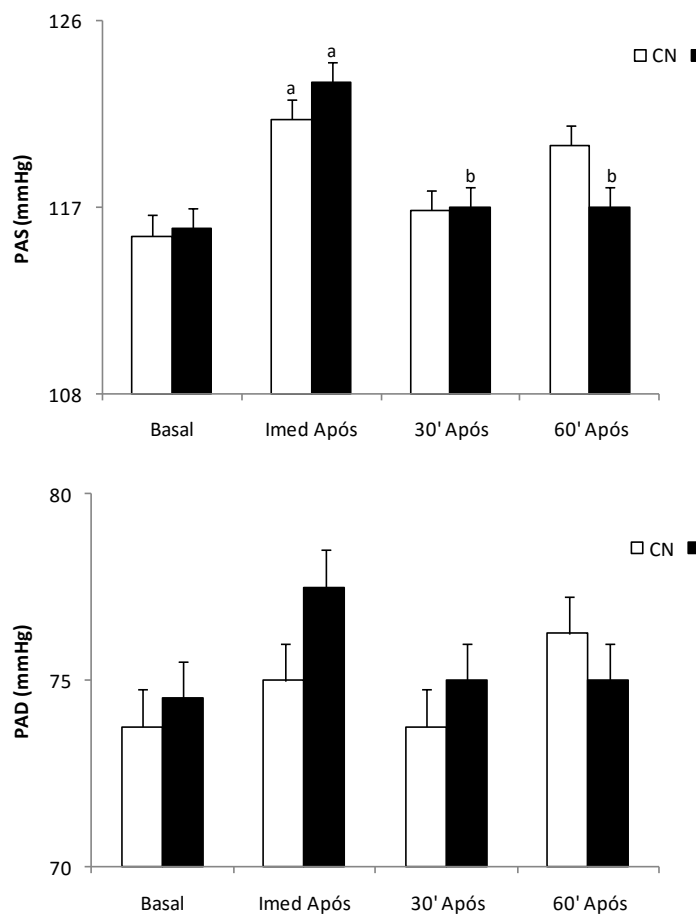

Figura 3. Resultados da Pressão Arterial. Nota: ANOVA two-way para medidas repetidas, com post-hoc utilizando Duncan Test. ${ }^{a}$ - diferente do basal, na mesma condição; ${ }^{b}$ - diferente do imediatamente após, na mesma condição. Resultados significativos para $p \leq 0.05$. Dados apresentados em média \pm desvio-padrão. Legenda: PAS pressão arterial sistólica; PAD - pressão arterial diastólica. Dados referentes a 10 voluntários.

A frequência cardíaca basal na condição de normóxia foi de $76.91 \pm 1.01 \mathrm{bpm}$ e na condição de hipóxia foi de $80.60 \pm 1.37$ bpm. Já durante os 45 minutos de exercício físico a média da frequência cardíaca apresentou um aumento e foi de $163.57 \pm 14.11 \mathrm{bpm}$ na condição de normóxia e $160.24 \pm 11.41$ bpm na condição de hipóxia. No período pósexercício, houve uma redução da frequência cardíaca e a condição de normóxia apresentou média de $138.25 \pm 2.83 \mathrm{bpm}$ e a condição de hipóxia $139.76 \pm 2.95 \mathrm{bpm}$.

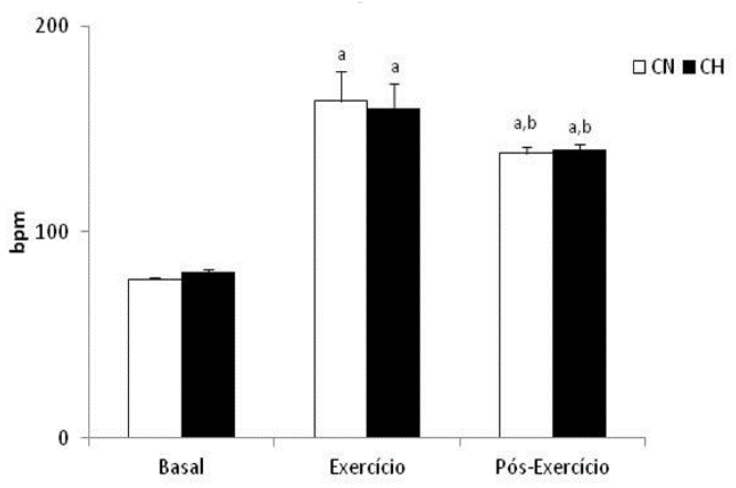

Figura 4 . Resultados da Frequência Cardíaca. Nota: ANOVA two-way para medidas repetidas, com posthoc utilizando Duncan Test. ${ }^{a}$ - diferente do basal, na mesma condição; ${ }^{b}$ - diferente do exercício, na mesma condição. Resultados significativos para $p \leq 0.05$. Dados apresentados em média \pm desvio-padrão.

\section{DISCUSSÃO}

O presente estudo mostrou que o exercício físico quando realizado na intensidade do LV-I durante 45 minutos em ambiente hipóxico equivalente a $4.500 \mathrm{~m}$, com diminuição na fração expirada de $\mathrm{O}_{2}$, piora o estado de humor de jovens saudáveis.

De forma inesperada e curiosa, a partir da análise das respostas afetivas, não foi observado diferenças entre os grupos quando as condições de normóxia e hipóxia foram comparadas. Era esperado que o estresse do exercício combinado ao estresse da hipóxia poderia representar uma piora no estado de humor quando comparado a condição de normóxia, mas o tempo de exposição à hipóxia, o número de participantes do estudo e as diferenças inter-individuais possam tem influenciado em tal resultado. No entanto, na condição de hipóxia, os voluntários apresentaram maiores escores de raiva, fadiga, confusão mental e DTH e diminuição do vigor de acordo com o instrumento BRUMS por um período de até 30 minutos após o cessamento do exercício, mesmo período em que os voluntários permaneceram em hipóxia. Tais resultados corroboram os achados de Lemos et al. (2010) que observaram comportamento similar em indivíduos expostos a altitude durante 24 horas. Em contrapartida, um grupo 
de alpinistas que testaram estratégias de ascensão a grandes altitudes com o intuito de estabelecer uma melhor aclimatação não apresentaram diferenças significativas em tarefas que avaliaram o humor e a cognição (Bonnon, Noël-Jorand, \& Therme, 1999).

De acordo com o instrumento SEES, após a prática do exercício físico na condição de hipóxia, os voluntários ficaram fadigados por um maior espaço de tempo e apresentaram maiores níveis de distresse psicológico, que significaria um aumento negativo do estresse, acompanhados de uma diminuição do bemestar, que pode estar intimamente relacionado as condições estressantes as quais os sujeitos foram submetidos. Essas dimensões tiveram o retorno dos scores aos valores basais somente 60 min. após o término do exercício, momento em que os questionários foram aplicados fora da câmara normobárica, sugerindo que estes valores podem estar relacionados com a condição de hipóxia a que os voluntários estavam mesmo com o cessamento do exercício. Utilizando o mesmo instrumento, Giampá et al. (2012) mostraram o mesmo comportamento das dimensões do referido questionário, porém os participantes realizaram exercício físico até a exaustão voluntária máxima, mostrando que a intensidade do exercício não é capaz de interferir em tais respostas.

A saturação funcional de oxigênio da hemoglobina arterial apresentou alterações na condição de hipóxia quando comparada com a de normóxia. Os valores da oximetria apontam uma diminuição imediata após a prática do exercício físico seguido de uma diminuição ainda maior $30 \mathrm{~min}$. após o término da atividade, sendo que ao nível do mar seus valores não apresentaram diferenças significativas. Porém, na condição de hipóxia, os escores de saturação funcional de oxigênio da hemoglobina arterial retornaram aos valores basais $60 \mathrm{~min}$. após o término do exercício. Isso provavelmente se deve ao fato de que os voluntários ficaram expostos a altitude simulada até $30 \mathrm{~min}$. após o término do exercício. Esta foi a única variável que apresentou diferença intergrupo, sugerindo- nos que esta sofre grande influência das características presentes num ambiente hipóxico. Assim como em nosso estudo, Mollard et al. (2007) demonstraram uma maior redução da saturação funcional de oxigênio da hemoglobina arterial em seu estudo, que consistia em um grupo de indivíduos treinados que realizaram um teste máximo de carga incremental em um cicloergômetro de membro inferior, na condição de hipóxia simulada referente a 5 altitudes: $1000 \mathrm{~m}, 1500 \mathrm{~m}, 2500 \mathrm{~m}$, $3500 \mathrm{~m}$ e $4500 \mathrm{~m}$ quando comparado ao grupo de indivíduos não treinados. $O$ grupo conseguiu demonstrar que a saturação funcional de oxigênio da hemoglobina arterial é um forte preditor do $\dot{V} \mathrm{O}_{2}$ máximo. Para eles, a principal explicação é a limitação de difusão alvéolo-capilar que resulta do aumento do débito cardíaco, reduzindo o tempo de trânsito da célula vermelha do sangue em capilares pulmonares. Entretanto, em normóxia, a limitação de difusão aparece para um nível importante de $\dot{V} \mathrm{O}_{2}$.

Em relação a variável pressão arterial, tanto a sistólica como a diastólica apresentaram comportamento similar em ambas as condições experimentais, onde é possível observar um aumento após a realização do exercício da pressão arterial sistólica e nenhuma diferença significativa na pressão arterial diastólica. Tal comportamento já está bem descrito na literatura, onde podemos encontrar que o exercício aeróbio praticado por jovens saudáveis produz um aumento na pressão arterial sistólica, sem alterações na pressão arterial diastólica imediatamente após o término da atividade. Cessado o exercício físico, é amplamente elucidado que este possui um efeito hipotensor após a sua realização, com conseqüente diminuição da pressão arterial sistólica (Duncan et al., 2014), comportamento que também foi observado em nosso estudo, onde verificamos que no instante $30 \mathrm{~min}$ após o término do exercício físico os valores de pressão arterial sistólica foram similares ao instante que antecedeu o exercício.

Esta adaptação cardiovascular em resposta ao exercício físico faz também com que a 
frequência cardíaca seja maior durante a atividade, já que a necessidade de um maior aporte energético para a musculatura esquelética promove um aumento do número de batimentos por minuto ( $\mathrm{Pal}$, RadavelliBagatini, \& Ho, 2013). Tanto na condição de normóxia quanto de hipóxia o comportamento da frequência cardíaca foi o mesmo, onde a média durante o exercício foi maior quando comparado ao período basal e pós exercício. Embora não tenhamos encontrado diferenças significativas entre as condições, vale ressaltar que a intensidade do exercício na condição de hipóxia foi diminuída, e mesmo assim a média da frequência cardíaca durante o exercício foi igual ao da condição de hipóxia. Em resposta a exposição a elevada altitude, visando uma maior adaptação ao déficit na utilização do oxigênio, há um aumento da síntese de catecolaminas - principalmente a noradrenalina. $\mathrm{O}$ aumento da função simpática acelera a freqüência cardíaca e aprimora a contratilidade do miocárdio, aumentando assim o fluxo sangüíneo para os músculos, o consumo de oxigênio e a mobilização de glicogênio, concomitantemente ao aumento da pressão arterial (Faria et al., 2005). Além disso, por se tratar de uma amostra composta por jovens do sexo masculino, o efeito vasodilatador do exercício realizado em hipóxia é menor quando comparado com jovens do sexo feminino, devido a uma menor ativação dos receptores $\beta$-adrenérgicos (Casey, Shepherd, \& Joyner, 2014), refletindo no aumento da pressão arterial sistólica.

O comportamento das catecolaminas, além de influenciarem nas adaptações fisiológicas frente ao estresse da hipóxia, podem interferir no estado de humor. Ray et al. (2011) verificaram que a exposição a hipóxia por um período de 7 dias resultou em um aumento de noradrenalina e dopamina no córtex frontal, no tronco cerebral, no cerebelo e na ponte, com concomitante diminuição dos níveis de serotonina no córtex frontal. Embora este estudo apresente resultados após uma semana de exposição a hipóxia, a exposição aguda a este ambiente, somando-se a prática do exercício físico, são os responsáveis por alterar a síntese desses neurotransmissores em resposta a diminuição da fração expirada de oxigênio e tal fato pode explicar as alterações fisiológicas como o aumento da pressão arterial sistólica - e as alterações psicológicas - com a piora no estado de humor.

\section{CONCLUSÕES}

Conclui-se que o exercício físico moderado aumenta a pressão arterial sistólica tanto em normóxia quanto em hipóxia, no entanto quando realizado em condição de altitude simulada de $4500 \mathrm{~m}$ promove uma piora no estado de humor de jovens saudáveis. Além disto, o tempo da prática do exercício físico, o número de voluntários e o tempo de exposição a hipóxia simulada e a diminuição da intensidade do exercício físico na condição hipóxia podem ter sido um fator limitante na detecção de mais diferenças entre as dimensões estudadas. Mais estudos a respeito da temática são desejáveis a fim de investigar os mecanismos envolvidos e visando estabelecer melhores estratégias que minimizem tais efeitos.

\begin{tabular}{l}
\hline Agradecimentos: \\
Os autores agradecem o apoio científico de Everald \\
Vancouler e o apoio técnico e financeiro da AFIP e \\
do CEPE.
\end{tabular}

Conflito de Interesses:

Nada a declarar.

Financiamento:

Fapesp, Cnpq, Capes.

\section{REFERÊNCIAS}

Ando, S., Hatamoto, Y., Sudo, M., Kiyonaga, A., Tanaka, H., \& Higaki, Y. (2013). The effects of exercise under hypoxia on cognitive function. Plos One, 8(5), e63630. http://doi.org/10.1371/journal.pone.0063630

Ando, S., Yamada, Y., \& Kokubu, M. (2010). Reaction time to peripheral visual stimuli during exercise under hypoxia. Journal of Applied Physiology (Bethesda, Md.: 1985), 108(5),

1210-1216. http://doi.org/10.1152/japplphysiol.01115.20 09

Araújo, R. C. (2009). Efeitos da exposição à altitude no desempenho físico. EFDeportes.com, 
80 | JFT Souza, SQC Giampá, VA Lemos, RVT Santos, MT Mello, HKM Antunes

Revista Digital, (129). Retrieved from http://www.efdeportes.com/efd129/efeitosda-exposicao-a-altitude-no-desempenhofisico.htm

Bahrke, M. S., \& Shukitt-Hale, B. (1993). Effects of altitude on mood, behaviour and cognitive functioning. A review. Sports Medicine, 16(2), 97-125.

Bonnon, M., Noël-Jorand, M. C., \& Therme, P. (1999). Criteria for psychological adaptation to high-altitude hypoxia. Perceptual and Motor Skills, $\quad$ 89(1), 3-18. http://doi.org/10.2466/pms.1999.89.1.3

Born, D. P., Hoppe, M. W., Lindner, N., Freiwald, J., Holmberg, H.-C., \& Sperlich, B. (2014). [Adaptive mechanisms and behavioural recommendations: playing football in heat, cold and high altitude conditions]. Sportverletzung Sportschaden, 28(1), 17-23. http://doi.org/10.1055/s-0034-1366055

Casey, D. P., Shepherd, J. R. A., \& Joyner, M. J. (2014). Sex and vasodilator responses to hypoxia at rest and during exercise. Journal of Applied Physiology (Bethesda, Md.: 1985), 116(7), 927-936. http://doi.org/10.1152/japplphysiol.00409.20 13

Chapman, R. F., Stickford, J. L., \& Levine, B. D. (2010). Altitude training considerations for the winter sport athlete. Experimental Physiology, 95(3), 411-421. http://doi.org/10.1113/expphysiol.2009.0503 77

de Aquino Lemos, V., Antunes, H. K. M., dos Santos, R. V. T., Lira, F. S., Tufik, S., \& de Mello, M. T. (2012). High altitude exposure impairs sleep patterns, mood, and cognitive functions. Psychophysiology, 49(9), 1298-1306. http://doi.org/10.1111/j.14698986.2012.01411.x

Duncan, M. J., Clarke, N. D., Birch, S. L., Tallis, J., Hankey, J., Bryant, E., \& Eyre, E. L. J. (2014). The Effect of Green Exercise on Blood Pressure, Heart Rate and Mood State in Primary School Children. International Journal of Environmental Research and Public Health, 11(4), 3678-3688. http://doi.org/10.3390/ijerph110403678

Faria, A. P., Dehn, A. L., Lala Filho, E. L., Assis, G. M., Pinto, P. R. B., Salvi, R. C., \& Campos Filho, V. D. (2005). Treinamento na altitude. Movimento \& Percepção, 5(6), 150-162.

Giampá, S. Q. de C., Souza, J. F. T. de, Santos, S. A. dos, Silva, E. T. da, Mello, M. T. de, Santos, R. V. T., \& Antunes, H. K. M. (2012). Respostas Afetivas do Exercício Físico Realizado até Exaustão Voluntária Máxima em Condição de Hipóxia Simulada. Revista Psicologia E Saúde, 4(1), 19-26.

Jones, A. M., \& Doust, J. H. (1996). A 1\% treadmill grade most accurately reflects the energetic cost of outdoor running. Journal of Sports
Sciences, $\quad$ 14(4), 321-327.

http://doi.org/10.1080/02640419608727717

Lemos, V. de A., Antunes, H. K. M., Santos, R. V. T. dos, Prado, J. M. da S., Tufik, S., \& Mello, M. T. D. (2010). Efeitos da exposição à altitude sobre os aspectos neuropsicológicos: uma revisão da literatura. Revista Brasileira de Psiquiatria, 32(1), 70-76. http://doi.org/10.1590/S151644462009005000013

Lox, C. L., \& Rudolph, D. L. (1994). The Subjective Exercise Experiences Scale (SEES): Factorial validity and effects of acute exercise. Journal of Social Behavior \& Personality, 9(4), 837-844.

Mannie, Z. N., Williams, C., Diesch, J., Steptoe, A., Leeson, P., \& Cowen, P. J. (2013). Cardiovascular and metabolic risk profile in young people at familial risk of depression. The British Journal of Psychiatry: The Journal of Mental Science, 203(1), 18-23. http://doi.org/10.1192/bjp.bp.113.126987

Mazzeo, R. S. (2005). Altitude, exercise and immune function. Exercise Immunology Review, 11, 616.

McAuley, E., \& Courneya, K. S. (1994). The Subjective Exercise Experience Scale (SEES): development and preliminary validation. Journal of Sport and Exercise Psychology, 16(2), 163-177.

McNair, D. M., Lorr, M., \& Droppleman, L. F. (1971). EITS Manual for the Profile of Mood States (1st ed.). San Diego, CA, US: Educational and Industrial Testing Service.

Mollard, P., Woorons, X., Letournel, M., Cornolo, J., Lamberto, C., Beaudry, M., \& Richalet, J.-P. (2007). Role of maximal heart rate and arterial $\mathrm{O} 2$ saturation on the decrement of $\mathrm{VO} 2 \mathrm{max}$ in moderate acute hypoxia in trained and untrained men. International Journal of Sports Medicine, 28(3), 186-192. http://doi.org/10.1055/s-2006-924215

Nelson, M. E., Rejeski, W. J., Blair, S. N., Duncan, P. W., Judge, J. O., King, A. C., ... American Heart Association. (2007). Physical activity and public health in older adults: recommendation from the American College of Sports Medicine and the American Heart Association. Circulation, 116(9), 1094-1105. http://doi.org/10.1161/CIRCULATIONAHA. 107.185650

Pal, S., Radavelli-Bagatini, S., \& Ho, S. (2013). Potential benefits of exercise on blood pressure and vascular function. Journal of the American Society of Hypertension, 7(6), 494-506. http://doi.org/10.1016/j.jash.2013.07.004

Peng, Q., Basang, Z., Cui, C., Li, L., Qian, J., Gesang, Q., ... Jin, L. (2013). Physiological Responses and Evaluation of Effects of BMI, Smoking and Drinking in High Altitude Acclimatization: A Cohort Study in Chinese Han Young Males. PLOS ONE, 8(11), e79346. http://doi.org/10.1371/journal.pone.0079346 
Pointer, M. A., Livingston, J. N., Yancey, S., McClelland, M. K., \& Bukoski, R. D. (2008). Psychosocial factors contribute to resting blood pressure in African Americans. Ethnicity \& Disease, 18(3), 289-293.

Ray, K., Dutta, A., Panjwani, U., Thakur, L., Anand, J. P., \& Kumar, S. (2011). Hypobaric hypoxia modulates brain biogenic amines and disturbs sleep architecture. Neurochemistry International, $\quad 58(1)$, 112-118. http://doi.org/10.1016/j.neuint.2010.11.003

Rohlfs, I. C. P. de M., Rotta, T. M., Luft, C. D. B., Andrade, A., Krebs, R. J., \& Carvalho, T. de. (2008). A Escala de Humor de Brunel (Brums): instrumento para detecção precoce da síndrome do excesso de treinamento. Revista Brasileira de Medicina Do Esporte, 14(3), 176181. http://doi.org/10.1590/S151786922008000300003

Sharma, V. K., Das, S. K., Dhar, P., Hota, K. B., Mahapatra, B. B., Vashishtha, V., ... Srivastava, R. B. (2014). Domain specific changes in cognition at high altitude and its correlation with hyperhomocysteinemia. PloS One, 9(7), e101448. http://doi.org/10.1371/journal.pone.0101448

Subudhi, A. W., Dimmen, A. C., \& Roach, R. C. (2007). Effects of acute hypoxia on cerebral and muscle oxygenation during incremental exercise. Journal of Applied Physiology (Bethesda, Md.: 1985), 103(1), 177-183. http://doi.org/10.1152/japplphysiol.01460.20 06

Subudhi, A. W., Miramon, B. R., Granger, M. E., \& Roach, R. C. (2009). Frontal and motor cortex oxygenation during maximal exercise in normoxia and hypoxia. Journal of Applied Physiology (Bethesda, Md.: 1985), 106(4), 1153-1158.

http://doi.org/10.1152/japplphysiol.91475.20 08

Terry, P. C., Lane, A. M., \& Fogarty, G. J. (2003). Construct validity of the profile of mood states - adolescents for use with adults. Psychology of Sport and Exercise, 4(2), 125-139.

Virués-Ortega, J., Garrido, E., Javierre, C., \& Kloezeman, K. C. (2006). Human behaviour and development under high-altitude conditions. Developmental Science, 9(4), 400$410 . \quad$ http://doi.org/10.1111/j.14677687.2006.00505.x

Walsh, A. L., Gonzalez, A. M., Ratamess, N. A., Kang, J., \& Hoffman, J. R. (2010). Improved time to exhaustion following ingestion of the energy drink Amino Impact ${ }^{\mathrm{TM}}$. Journal of the International Society of Sports Nutrition, 7, 14. http://doi.org/10.1186/1550-2783-7-14 